\title{
H.264 Layered Coded Video over Wireless Networks: Channel Coding and Modulation Constraints
}

\author{
M. M. Ghandi, B. Barmada, E. V. Jones, and M. Ghanbari \\ Department of Electronic Systems Engineering, University of Essex, Wivenhoe Park, Colchester CO4 3SQ, UK
}

Received 13 July 2005; Revised 16 December 2005; Accepted 18 February 2006

\begin{abstract}
This paper considers the prioritised transmission of H.264 layered coded video over wireless channels. For appropriate protection of video data, methods such as prioritised forward error correction coding (FEC) or hierarchical quadrature amplitude modulation (HQAM) can be employed, but each imposes system constraints. FEC provides good protection but at the price of a high overhead and complexity. HQAM is less complex and does not introduce any overhead, but permits only fixed data ratios between the priority layers. Such constraints are analysed and practical solutions are proposed for layered transmission of data-partitioned and SNR-scalable coded video where combinations of HQAM and FEC are used to exploit the advantages of both coding methods. Simulation results show that the flexibility of SNR scalability and absence of picture drift imply that SNR scalability as modelled is superior to data partitioning in such applications.
\end{abstract}

Copyright (๑) 2006 Hindawi Publishing Corporation. All rights reserved.

\section{INTRODUCTION}

Within a given bandwidth, the capacity of a communication channel is determined by its signal-to-noise ratio (SNR) [1] which can vary widely. Ideally, a service such as video over wireless networks should adaptively change its information rate according to the available channel capacity. For example, low SNRs can only support a low source rate and require a high protection of contents, and conversely for high SNRs, a high source rate can be transmitted with less protection [2]. However, this ideal adaptation is not feasible in many applications where the transmitter has no knowledge of the channel conditions such as in video broadcasting. The solution might be a conservative design which only considers low SNRs and so would have a low throughput at high SNRs. Alternatively, unequal error protection (UEP) has been proposed in which only an essential portion of the source contents are protected for low SNRs and the rest would be available only at higher SNRs $[3,4]$.

To achieve UEP for a video service, two distinct considerations are required. First, the contents of the coded video should be divided into layers that classify their importance. This can be achieved, for example, by the data partitioning of the H.264 standard ${ }^{1}$ [5]. Secondly, the network should offer a different protection against noise for each layer. Let

\footnotetext{
${ }^{1}$ H.264 is also called MPEG-4 part 10 advanced video coding (AVC), but throughout this paper for convenience we call it H.264.
}

us assume there is a high-priority (HP) and a low-priority (LP) layer with the corresponding source bit rates $s_{\mathrm{HP}}$ and $s_{\mathrm{LP}}$. One solution to achieve UEP is to incorporate prioritised forward error correction codes (FEC) with coding ratios $R_{\mathrm{HP}}<R_{\mathrm{LP}}$ which means greater coding protection for the HP layer. Hence, the total required channel rate (the sum of HP and LP channel rates) becomes

$$
\mathrm{ch}=\mathrm{ch}_{\mathrm{HP}}+\mathrm{ch}_{\mathrm{LP}}=\frac{s_{\mathrm{HP}}}{R_{\mathrm{HP}}}+\frac{s_{\mathrm{LP}}}{R_{\mathrm{LP}}} .
$$

Assuming the unequal channel-coding ratios, $R_{\mathrm{HP}}$ and $R_{\mathrm{LP}}$, are constant, then in order to have a constant total channel rate $(\mathrm{ch})$, not only the total source rate $\left(s_{\mathrm{HP}}+s_{\mathrm{LP}}\right)$ should be fixed, but also the allocation between $s_{\mathrm{HP}}$ and $s_{\mathrm{LP}}$ needs to be constant. In other words, both source bit rates $s_{\mathrm{HP}}$ and $s_{\mathrm{LP}}$ should be at constant rates-a condition which is not met in the case of data partitioning.

Another alternative for UEP is hierarchical quadrature amplitude modulation (HQAM) [6] in which the two most significant bits (MSBs) of each transmitted symbol with a gray mapping have a better immunity against the noise than the remaining least significant bits (LSBs). Although HQAM does not impose as much complexity on the system as FEC does, it is more limiting in that the fixed number of MSBs and LSBs requires $\mathrm{ch}_{\mathrm{HP}}$ and $\mathrm{ch}_{\mathrm{LP}}$ to be constant.

In this paper, practical solutions for UEP transmission of H.264 bitstreams are presented where we combine HQAM and FEC to take advantage of both. First for H.264 with data 


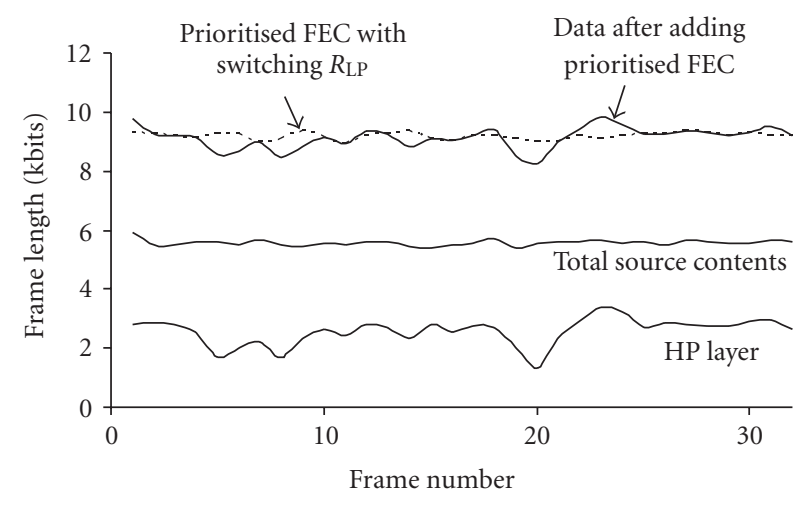

Figure 1: A constant rate data-partitioned video (48 kbps) after adding UEP (for Foreman QCIF test sequence at $10 \mathrm{~Hz}$, with $R_{\mathrm{HP}}=$ $\left.1 / 2, R_{\mathrm{LP}}=3 / 4\right)$.

partitioning, since the proportion of the HP and LP source rates cannot be easily controlled, we consider switching the channel-coding ratios in a prioritised FEC scenario as explained in Section 2. We then employ our switched multilevel HQAM [7] and show that a combination of switched HQAM and fixed FEC results in a better performance. However, data partitioning still suffers from picture drift where receiving the HP layer alone can lead to the accumulation of errors in pictures. Hence, we consider a drift-free H.264 SNR-scalable solution [8] and analyse its practical limitations in Section 3. In the simulation results of Section 4 we show that the flexibility of SNR scalability is better able to withstand the network constraints and is superior to data partitioning in UEP scenarios.

\section{UNEQUAL ERROR PROTECTION WITH DATA PARTITIONING IN H.264}

\subsection{UEP-DP with switched prioritised FEC (switched turbo coding)}

In the data-partitioning mode of H.264, each slice is divided into three network abstraction layer (NAL) units. NAL-A, the most important unit, carries addressing and motion data and NAL-B and NAL-C carry the intra- and interresidual data. In this work, we consider NAL-A as the HP layer and group both NAL-B and NAL-C into the LP layer. By adjusting the quantisation parameter, one can control the overall source rate $\left(s_{\mathrm{HP}}+s_{\mathrm{LP}}\right)$ for an acceptable low-delay transmission. However, in a constant bit rate stream, the bit rate of the HP layer is still variable (Figure 1) under the influence of picture contents and the motion of objects. Therefore, after adding the protection bits to this layer, the total channel data rate (1) is still variable in spite of the efforts of the source rate control.

To maintain a constant channel rate in UEP-DP, the channel-coding ratios $\left(R_{\mathrm{HP}}\right.$ and $\left.R_{\mathrm{LP}}\right)$ should be frequently adjusted with respect to the size of the HP and LP layers. We note that the main priority must be the HP layer. Thus, we do not compromise its protection (we fix $R_{\mathrm{HP}}$ whatever the size of the HP layer) and only vary $R_{\mathrm{LP}}$ to maintain a fixed total channel rate. In this paper, we allocate $60 \%$ of each transmitted packet to the source data and $40 \%$ to the parity. Figure 2 depicts the different switching modes in our UEP-DP with their corresponding capacities for the HP and LP source data. When loading packets of each frame to a smoothing buffer, the actual percentage between the HP and LP source units is calculated and the appropriate mode from Figure 2 that offers the closest HP and LP ratios is selected. Note that the selected mode is reported to the receiver in order to perform the corresponding channel decoding procedure. This very low-rate control data can be transmitted reliably, and in this paper it is assumed to be error-free.

The above FEC approach has certain limitations. For example, its parity bit overhead is high such that with a limited channel rate, the source rate must be restricted to very low values. However, reducing the source rate in data-partitioned video will increase the proportion of the HP layer, as the motion information becomes the dominant part of the data. This further limits the system performance because the LP layer will have less opportunity for protection, that is, $R_{\mathrm{LP}}$ will be more often switched to $1 / 1$. To overcome this problem, we employ HQAM to offer prioritisation as discussed below.

\subsection{UEP-DP with combined FEC and switched HQAM}

A conventional square M-HQAM constellation [6] offers two levels of priority, where $M(\geq 16)$ denotes the number of signal points in the constellation. HP data bits occupy the two most significant bits of each point label while LP data occupies the remaining bits (i.e., 2 bits for 16- and 4 bits for 64 HQAM). Figure 3(a) shows such a constellation diagram for 2-level 64-HQAM, where the distances between quadrants ( $a$ in Figure 3(a)) and between points inside each quadrant $b$ are adjusted such that $a>b$, giving a distance factor $\alpha=a / b$. For a given average signal power, increasing the value of $\alpha$ increases the HP protection, but decreases the LP protection, thus providing a simple UEP. However, the fixed number of MSBs and LSBs requires the channel rates $\mathrm{ch}_{\mathrm{HP}}$ and $\mathrm{ch}_{\mathrm{LP}}$ to be constant and as noted earlier, for data partitioning there is no such constant relationship. We therefore resort to a multilevel HQAM to switch the HP and LP bit lengths as explained below.

In a multilevel HQAM [9] the constellation points are placed in such a way that groups of bits within the point label have similar degrees of protection as illustrated in the constellation diagram of Figure 3(b) for 3-level 64-HQAM. Two distance factors are now introduced $\alpha=a / b$, and $\beta=b / c$. The values of $\alpha$ and $\beta$ will determine the system "mode." Mode- 1 with $\alpha=\beta=1$, is a nonhierarchical QAM where all bits have the same immunity to noise and could be assigned to LP data. In mode- 2 , by setting $\alpha>1$ (and $\beta=1$ ) the conventional HQAM is achieved, that is, there are $2 \mathrm{HP}$ bits and 4 LP bits. Finally, mode- 3 with $\alpha=1$ and $\beta>1$, gives the first 4 bits a higher immunity than the last 2 bits. By switching between these three modes the percentage of HP bits can be changed between $0 \%, 33 \%$, and $66 \%$ but its 


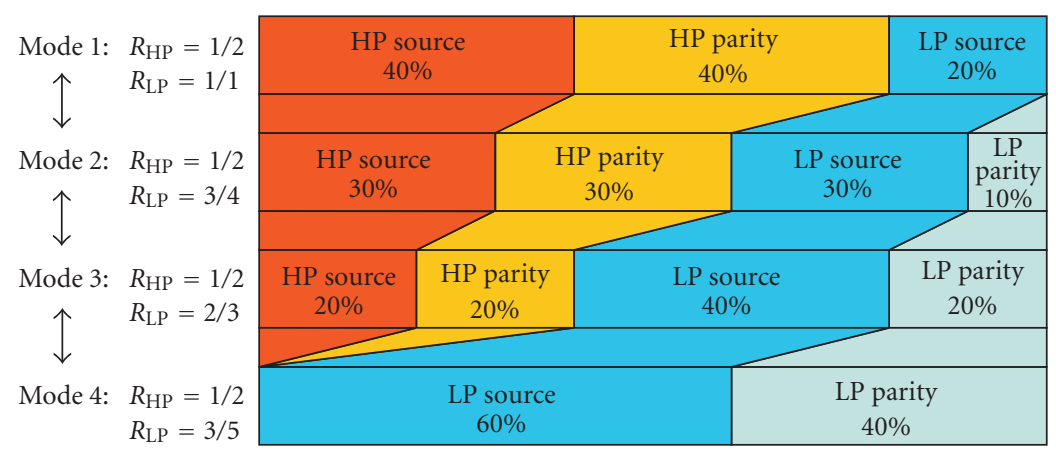

FIGURE 2: Capacity of a transmitted packet in switched turbo-coded UEP-DP.

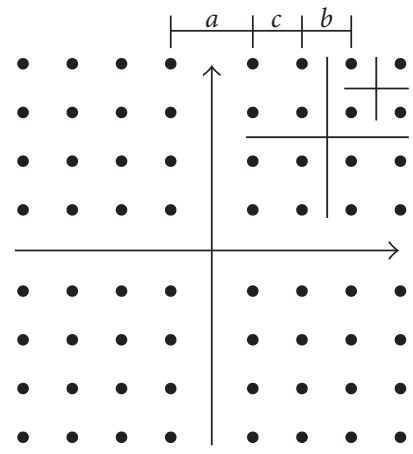

(a) Mode-2: $\alpha=a / b=1.5$, $\beta=b / c=1$

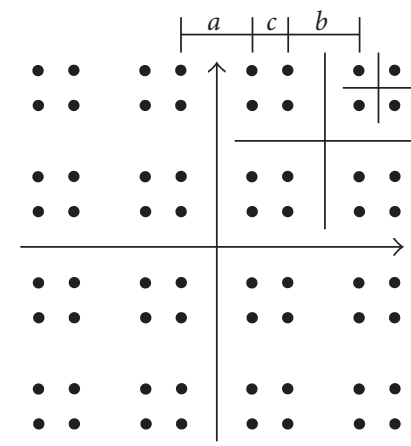

(b) Mode-3: $\alpha=a / b=1, \beta=$ $b / c=2$

FIgURE 3: Hierarchical constellations for 64-QAM.

protection remains unchanged as shown in Figure 4 with $\alpha$ and $\beta$ values as listed on the figure. What actually changes with this switching arrangement is the protection of the LP bits, similar to the switching of Section 2.1. For more details of this switched HQAM the readers are referred to [7].

The improved HP protection offered by HQAM is at the price of a lower noise immunity for the LP layer. In order to improve the protection of the layers, we can incorporate channel coding before modulation to shift the BER curves of Figure 4 towards the desired SNR region. This combination of switched HQAM and fixed FEC offers a number of advantages. Firstly, we can add protection with a constant channel-coding ratio for both HP and LP layers. Therefore, the LP data will never be transmitted unprotected, as opposed to the switched FEC where we often need to switch $R_{\mathrm{LP}}$ to $1 / 1$. Secondly, the protection of the HP layer becomes better than expected, as the high reliability of the HP bits soft information will improve the effectiveness of the turbo coding employed in this work. In simulation results we will show that this combination performs better than switched FEC. However, in the following we introduce another UEP solution with a proposed SNR scalability source coding arrangement which performs even better than the best effort UEP-DP.

\section{UNEQUAL ERROR PROTECTION FOR H.264 SNR SCALABILITY}

In this work we employ our H.264 SNR-scalable codec described in [8], which follows the general framework of SNR scalability defined in the standard video codecs [10]. The HP (base) layer of the scalable video is a fully standard compliant bitstream with a coarse quantisation step size while the LP (enhancement) layer contains additional data with a finer quantiser step size to enhance the video quality. Therefore, reception of the HP layer alone will give a drift-free service which is a desirable feature. Moreover, the existence of quantisation in both layers provides a flexibility to control the rates of the individual layers independently. Figure 5 shows the average HP source rate percentage for a wide range of total source rates from $10 \mathrm{kbps}$ to $200 \mathrm{kbps}$ ( $66 \%$ confidence limits, i.e., \pm one standard deviation are also shown). As we see in data partitioning, the portion of the bit rate assigned to the high-priority layer varies with the overall rate. That is why we need the complex adaptation described in Section 2. On the other hand with SNR scalability, as Figure 5 shows, over a wide range from 20 to $200 \mathrm{kbps}$ the required percentage for various network constraints can be easily met, with a reasonable confidence as indicated by small standard deviations. 


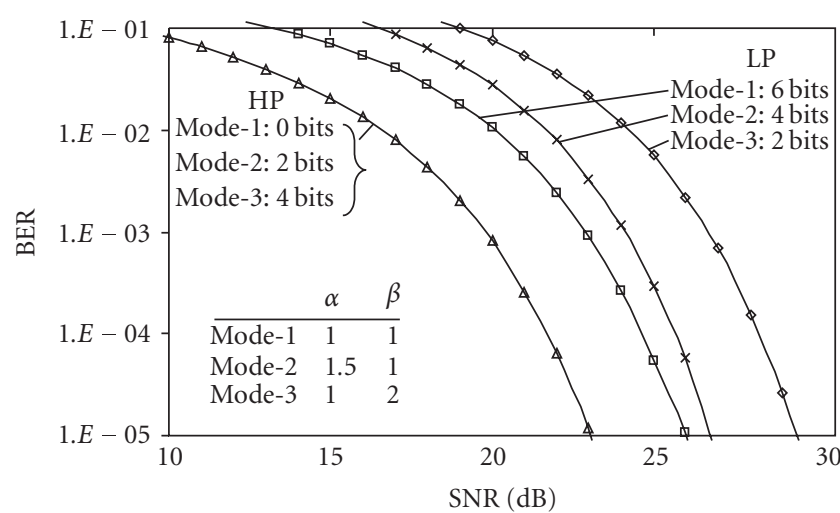

FIgURE 4: BER versus SNR for LP and HP bits for three HQAM modes.

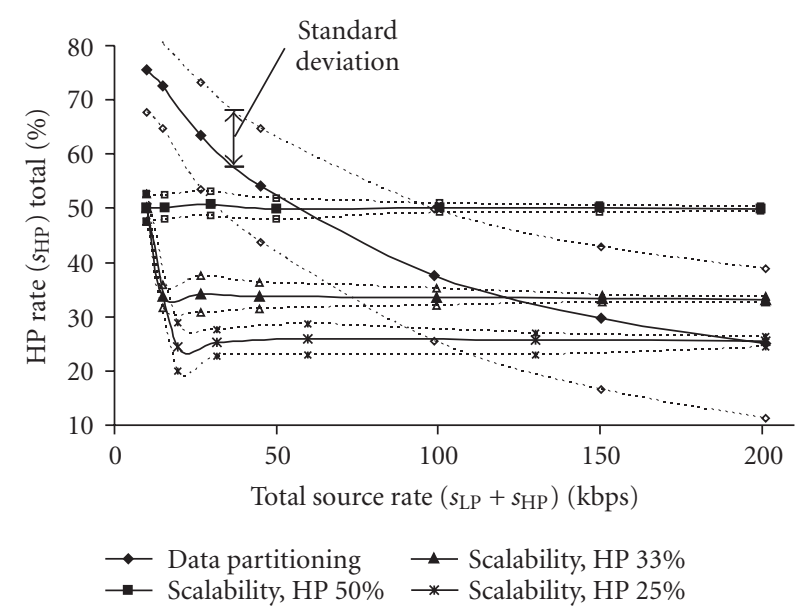

FIgURE 5: Mean HP source rate percentage (with \pm 1 standard deviation) versus total source rate, Foreman QCIF at $10 \mathrm{~Hz}$.

However, the drawback of SNR scalability is its higher overhead compared with data partitioning, resulting in lower picture quality for the same bit rate. This is shown in Figure 6 for a total source rate of $50 \mathrm{kbps}$. As can be seen, although scalability can offer a flexible range of HP percentages while data partitioning offers only one, the overhead has caused a drop in the total peak signal-to-noise ratio (PSNR) of up to $1 \mathrm{~dB}$. However, this penalty reduces with a lower HP percentage because the entropy coding of the enhancement layer improves as the data fraction reduces [8]. It should also be noted that it is generally desirable to keep the HP bit rate as low as possible. This is because lower HP rates contribute to a significantly lower overall channel rate on account of the FEC process and also reduce the average transmitter power of the HQAM. However, as Figure 6 shows, lowering the HP rate means a poorer HP quality and the rate and quality degradation of the base layer below $20 \%$ of the total rate is steep. On the other hand, an HP proportion above $40 \%$ means little contribution of the LP layer to the overall picture quality. Thus, we should limit the HP percentage to around $20 \%$ to $40 \%$ to ensure a balance between efficiency and quality. In

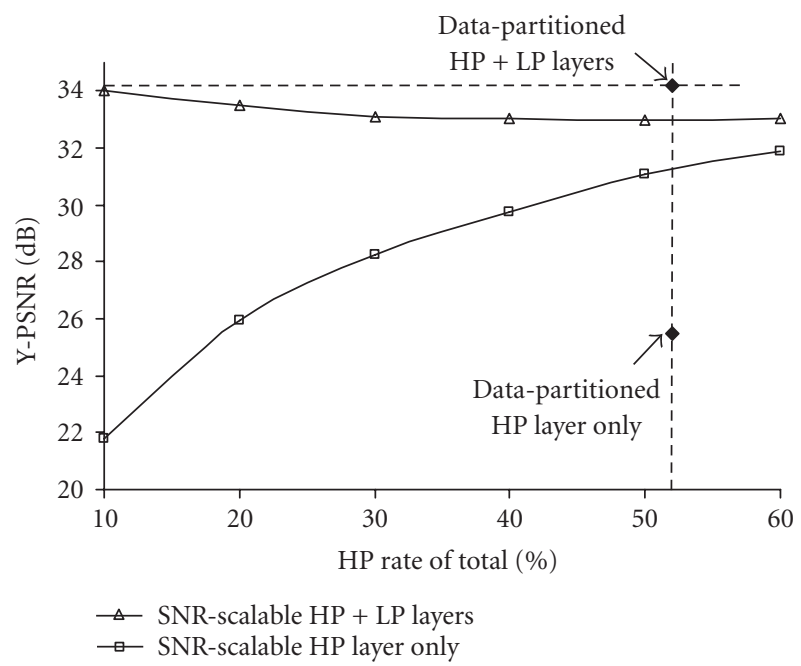

FIGURE 6: PSNR as a function of the proportion of HP source rate: $100 \times s_{\mathrm{HP}} /\left(s_{\mathrm{HP}}+s_{\mathrm{LP}}\right)$, Foreman QCIF at $10 \mathrm{~Hz}, s_{\mathrm{HP}}+s_{\mathrm{LP}}=50 \mathrm{kbps}$.

the following sections the incorporation of SNR scalability with UEP is discussed.

\subsection{UEP-SCAL with prioritised FEC}

Since the HP and LP source rates of the scalable video can be flexibly controlled, its unequal error protection does not require frequent switching as it does with data partitioning. Hence, fixed $R_{\mathrm{HP}}$ and $R_{\mathrm{LP}}$ values can be selected for the layered protection of contents. However, to select proper rates, different constraints do exist. As noted above, the proportion of the HP source rate $\left(s_{\mathrm{HP}}\right)$ should be within the region of good efficiency. Secondly, $R_{\mathrm{HP}}$ and $R_{\mathrm{LP}}$ should be determined such that the total channel rate (1) does not exceed the maximum available rate. These relationships are illustrated in Figure 7 for $R_{\mathrm{HP}}=1 / 3$ and $R_{\mathrm{LP}}=4 / 5$. It can be seen that only a limited region can be accepted as the practical adjustment between $s_{\mathrm{HP}}$ and $s_{\mathrm{LP}}$. However, even in this area, the rate of the HP layer is very low and will have a poor quality. One solution is to increase $R_{\mathrm{HP}}$ which means compromising HP protection. A better solution is to use HQAM which does not impose any overhead.

\subsection{UEP-SCAL with combined FEC and HQAM}

As mentioned in Section 2.2, the constraint on the HQAM is that its HP and LP capacities are constant. For example, for $64-\mathrm{HQAM}, \mathrm{ch}_{\mathrm{HP}}$ is $33 \%$ of the total channel rate. If there is no FEC, this will be the required percentage of the $s_{\mathrm{HP}}$ which is easily obtainable by our SNR-scalable codec. Therefore, for SNR scalability, we do not need to frequently change $\alpha$ and $\beta$ as in Section 2.2, so the use of conventional HQAM is sufficient. Thus, SNR-scalable video transmission with HQAM would be a simple and practical solution for many applications. The value of $\alpha$ simply determines how much distinction is made between the HP and LP protection [8]. 


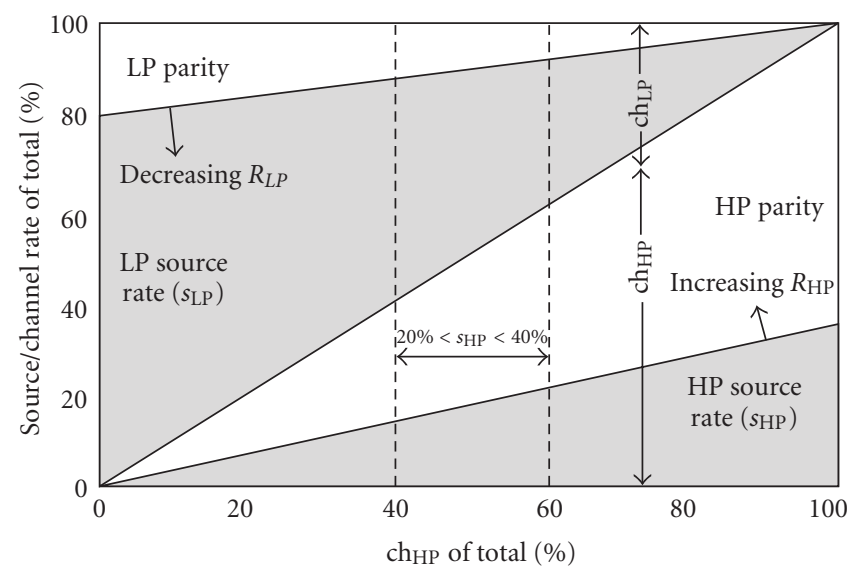

FIgURE 7: Source and channel rates for an FEC UEP scalable video with $R_{\mathrm{HP}}=1 / 3$ and $R_{\mathrm{LP}}=4 / 5$.

In some applications, the quality of the channel is so poor that there is no option but to add FEC to the coded source data. For a UEP scalable video using HQAM, we can add FEC with a single channel-coding ratio for both HP and LP layers and leave the task of UEP distinction completely to HQAM by adjusting $\alpha$. In this case $R_{\mathrm{HP}}=R_{\mathrm{LP}}$ and hence the source rate percentage remains unchanged.

Alternatively, we may wish to add different FEC to the two layers. In this case $R_{\mathrm{HP}} \neq R_{\mathrm{LP}}$, and the 64-HQAM limitation that $\mathrm{ch}_{\mathrm{HP}}$ should be $33 \%$ limits the flexibility of choices, that is, the source rates will be dictated to the codec by the selected $R_{\mathrm{HP}}$ and $R_{\mathrm{LP}}$ :

$$
s_{\mathrm{HP}}=0.33 \times \mathrm{ch} \times R_{\mathrm{HP}}, \quad s_{\mathrm{LP}}=0.66 \times \mathrm{ch} \times R_{\mathrm{LP}},
$$

where ch is the total available channel rate. Therefore, we should be careful that the HP rate percentage does not move outside the practical range. For a combined HQAM and FEC, we leave the task of UEP entirely to HQAM, which only changes levels of protection, leaving source and channel rates unchanged. As mentioned earlier, the combination of HQAM and turbo coding will add a protection to the HP layer that even the turbo coding alone with a lower channelcoding ratio cannot achieve. Therefore, for the same level of protection we can transmit more source information with this combination, than with turbo coding alone. This is evident from our simulation results.

\section{SIMULATION RESULTS}

The unequal-error-protected transmission of data-partitioned and SNR-scalable coded video have been simulated in a Gaussian channel as well as in a fading environment (COST 207 model [11]) with a constant total channel rate of $\mathrm{ch}=$ $100 \mathrm{kbps}$. For forward error correction we employed turbo codes with generators G1 $=5$ and G2 $=7$ and a Log-MAP algorithm with three iterations in the decoder. Other turbo coding (TC) parameters are the same as detailed in [12]. The received bits passed to the decoder include their reliabilities extracted from the soft demapping process for HQAM as in [13].
For all the tests, the Foreman QCIF sequence at $10 \mathrm{~Hz}$ is used with a total length of 33 frames comprising NALunits of no more than 150 bytes long. The first frame is an error-free intraframe and the rest are P-frames. The reason we did not consider more frames is that for data partitioning, the drift and so the average quality (which is the principle criterion in this paper) are directly related to the number of P-frames. We assumed that after 33 frames, an intraframe would stop the propagation of errors. For confidence, we ran each experiment 100 times and recorded the average results. We should mention that - although not demonstrated for brevity-these experiments have been repeated for the News video sequence and similar trends have been observed.

The PSNR of pictures versus channel symbol SNR is depicted in Figure 8 for two UEP-DP scenarios in a Gaussian and a fading channel. The source rate of the data-partitioned video for both cases is $60 \mathrm{kbps}$ while the remaining $40 \mathrm{kbps}$ of the channel rate is dedicated to the FEC codes. As a benchmark, three nonlayered cases are also included in the figure (shown dotted) with different source rates and channelcoding ratios as listed on the figure. It can be seen that our switched HQAM combined with fixed TC has outperformed the switched TC alone. For the HP part (low SNRs), it has provided a better protection even with a higher $R_{\mathrm{HP}}$, and for the LP part the advantage of the combined method is evident. Comparing Figures 8(a) and 8(b) it can be seen that higher channel SNR is required for the fading channel than for the Gaussian for the same service but the advantage of the combined method is evident.

Comparing the UEP-DP graphs with the nonlayered ones is also interesting. When the entire channel rate is dedicated to the source, that is, $s=100 \mathrm{kbps}$ and $R=1 / 1$, the service will be available only at high SNRs and UEP-DP is clearly a more attractive choice. By comparing the combined HQAM and TC and the nonlayered graph at $60 \mathrm{kbps}$ (the same source rate), it can be observed that the UEP-DP has a lower performance than the nonlayered curve in some SNR regions of the Gaussian channel. However, surprisingly in a fading channel it has outperformed the nonlayered curve at all SNR regions (except its negligible overhead at very high SNR). This 


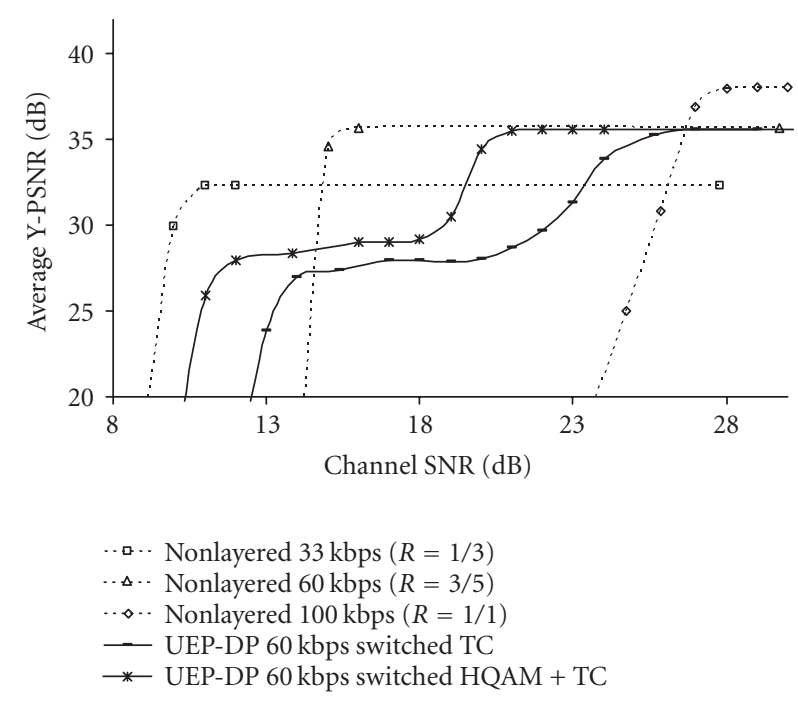

(a) In a Gaussian channel

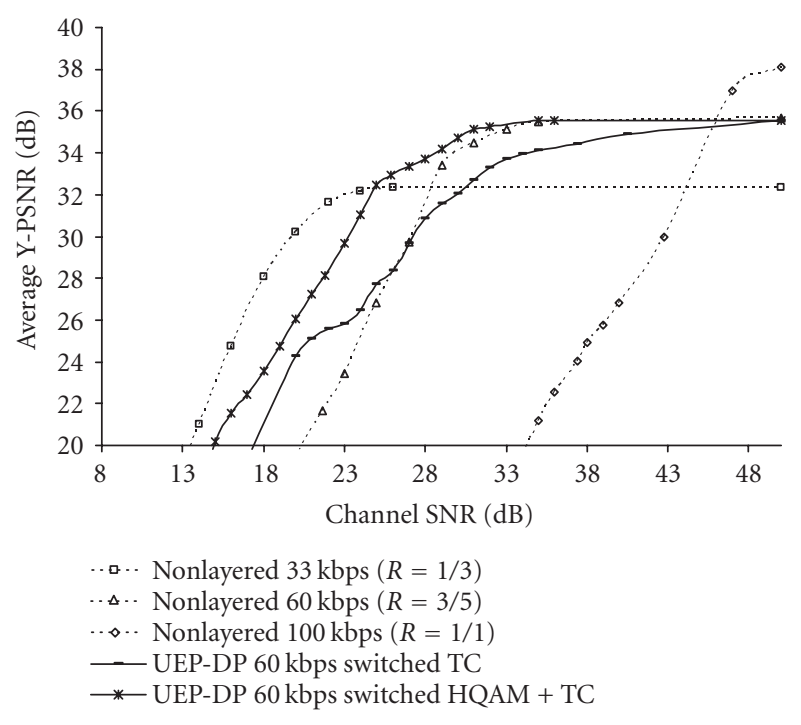

(b) In a fading channel

FIGURE 8: Foreman QCIF at $10 \mathrm{~Hz}$, UEP-DP $s_{\mathrm{HP}}+s_{\mathrm{LP}}=60 \mathrm{kbps}$, with switched TC: $R_{\mathrm{HP}}=1 / 2, R_{\mathrm{LP}}=\{3 / 5,2 / 3,3 / 4$, and $1 / 1\}$, and with switched HQAM combined with fixed TC: $R_{\mathrm{HP}}=R_{\mathrm{LP}}=3 / 5$.

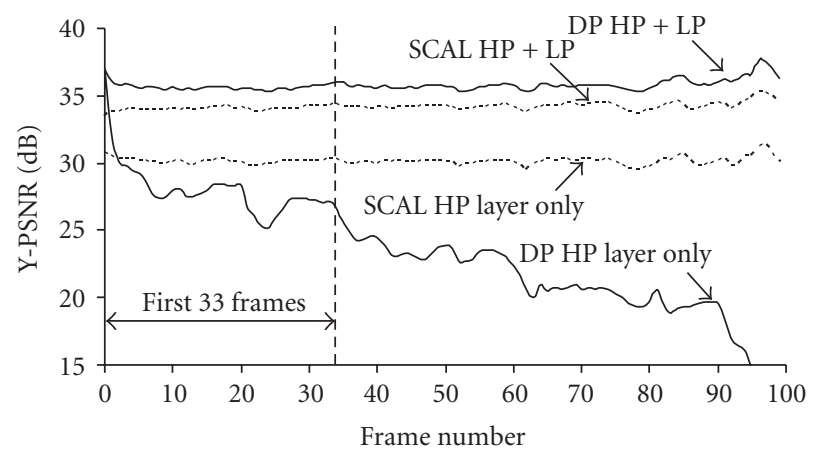

FIGURE 9: Error-free frame-by-frame PSNR, 10 seconds of Foreman QCIF@10 Hz, data-partitioned (DP): $s_{\mathrm{HP}}+s_{\mathrm{LP}}=60 \mathrm{kbps}$, and SNR scalable $(\mathrm{SCAL}): s_{\mathrm{HP}}=20 \mathrm{kbps}, s_{\mathrm{LP}}=40 \mathrm{kbps}$.

is because turbo coding in the fading channel does not perform as good as in the Gaussian channel. However, in a conservative design, by dedicating $66 \mathrm{kbps}$ of the channel rate to the FEC ( $s=33 \mathrm{kbps}, R=1 / 3)$ a video service is available over a wide SNR range with even a better quality than UEP-DP at the lower SNRs. This is the price to pay for unequal error protection with data partitioning, where much of this degradation is the result of picture drift. In fact, if more than 33 consecutive P-frames had been selected, the average PSNRs of UEP-DP would have been even worse. This can be observed from Figure 9 where it is clear that even the errorfree reception of the HP layer alone for data partitioning does not provide a stable picture quality.

However, the dotted plot in Figure 9 shows that SNR scalability does not suffer from picture drift, so we can ex- pect better results from UEP-SCAL especially because the channel-coding ratios are fixed. Figure 10 demonstrates the average PSNRs for UEP-SCAL with turbo coding alone $\left(R_{\mathrm{HP}}=1 / 3, R_{\mathrm{LP}}=4 / 5\right)$ and with a combined HQAM and TC $\left(R_{\mathrm{HP}}=R_{\mathrm{LP}}=3 / 5, \alpha=1.5\right)$. The advantage of our combined method is evident from the figure; it allows a higher $s_{\mathrm{HP}}$ for yet a better HP protection. Comparing with the conservative nonlayered curve $(R=1 / 3)$ at low SNRs, the UEPSCAL with the combined method has offered a video service with somewhat less quality. However, at the other extreme for higher SNRs, it gives more than $2 \mathrm{~dB}$ improvement on the video quality. This is the desired graceful service characteristic of a layered codec.

Figure 11 now compares the best effort data partitioned method (UEP-DP) of Figure 8 with the scalability method 


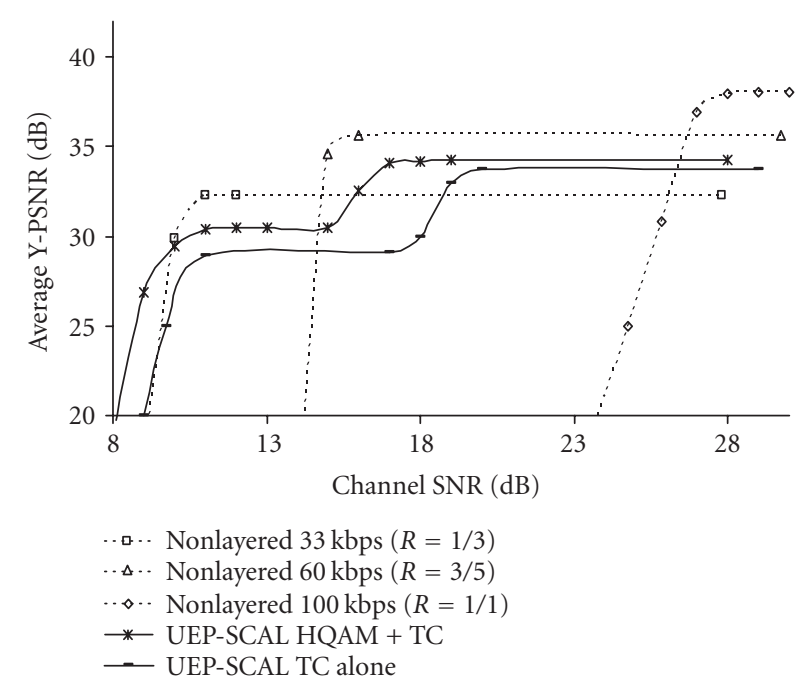

(a) In a Gaussian channel

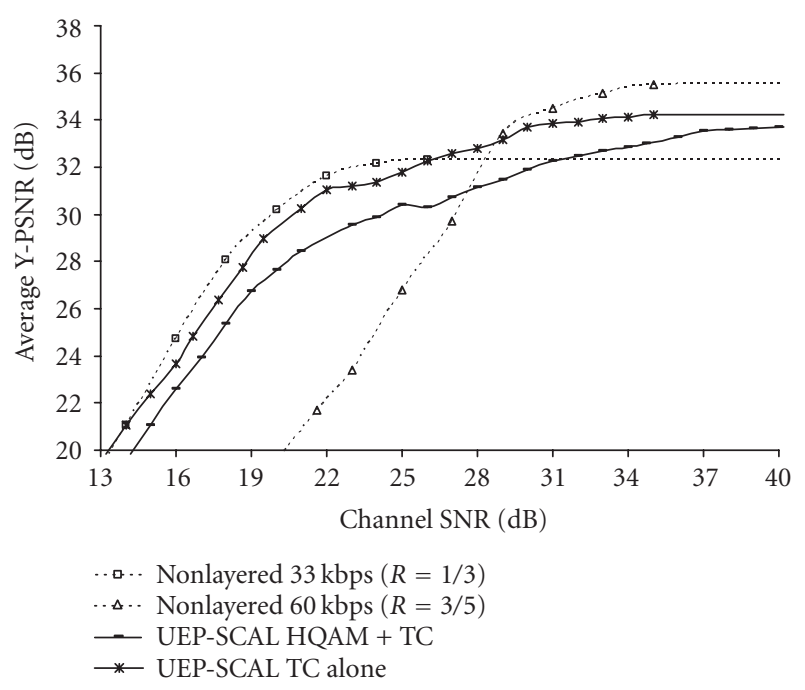

(b) In a fading channel

FIGURE 10: Foreman QCIF@10 Hz, UEP-SCAL with turbo coding alone: $s_{\mathrm{HP}}=16.6 \mathrm{kbps}, R_{\mathrm{HP}}=1 / 3, s_{\mathrm{LP}}=40 \mathrm{kbps}, R_{\mathrm{LP}}=4 / 5$, and combined HQAM and turbo coding: $s_{\mathrm{HP}}=20 \mathrm{kbps}, s_{\mathrm{LP}}=40 \mathrm{kbps}, R_{\mathrm{HP}}=R_{\mathrm{LP}}=3 / 5, \alpha=1.5$.

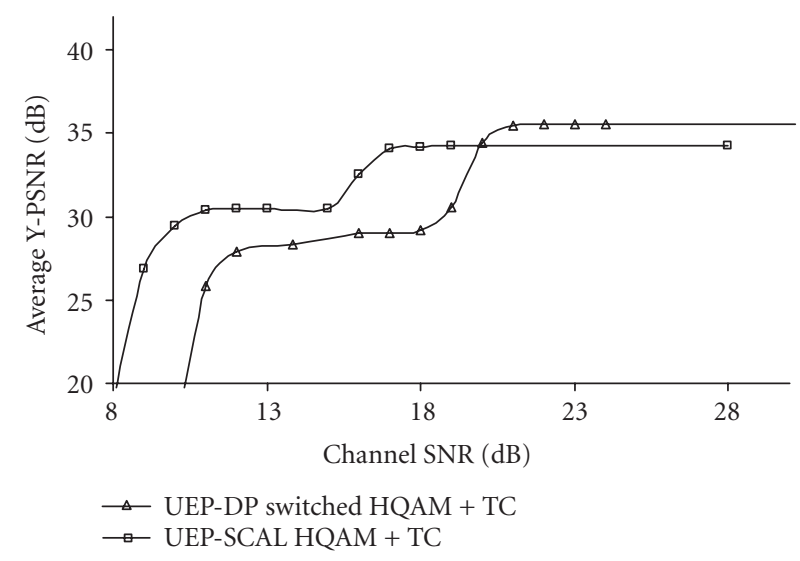

(a) In a Gaussian channel

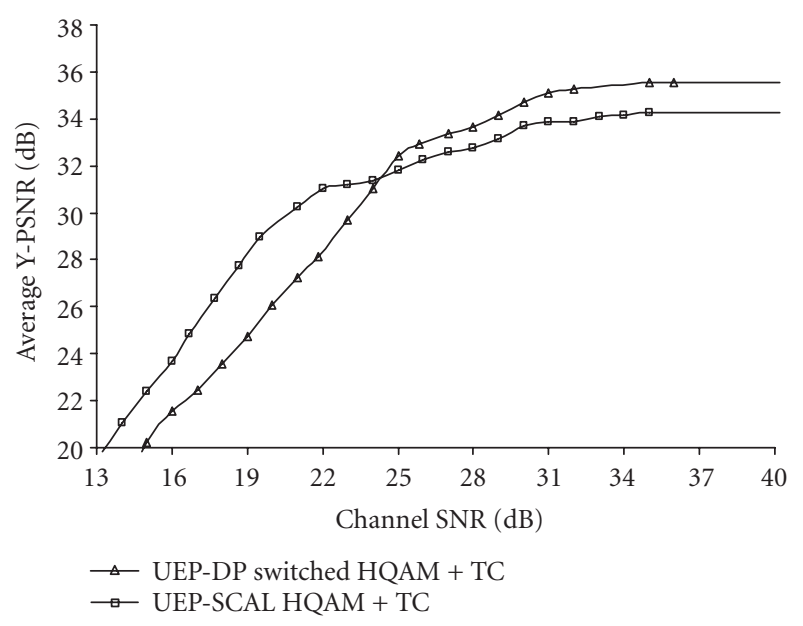

(b) In a fading channel

Figure 11: Best effort UEP-DP and UEP-SCAL, selected from Figures 8 and 10.

(UEP-SCAL) of Figure 10. For both Gaussian and fading channels, it can be seen that SNR scalability has clearly outperformed the best effort UEP-DP at lower SNRs with a relatively small penalty at high SNRs as a result of its overhead. As explained earlier this superiority has two explanations: SNR scalability does not suffer from picture drift, and secondly, it can flexibly cope with the constraints imposed by the hierarchical QAM.

\section{CONCLUSION}

We have shown that by combining HQAM and turbo coding, a more effective unequal-error-protected video transmission system can be achieved. However, the conventional HQAM imposes a severe constraint such that the bit rates of all layers need to be controlled. This is not met by data partitioning but can be achieved with SNR scalability as well as with any scalability that can control the bit rate of the layers. Since the current specification of H.264 can only support data partitioning at a given temporal resolution, we have suggested a switched HQAM that can cope with the resulting variable layers bit rate ratio. However, the simulation results showed that SNR scalability can still be superior to data partitioning in an unequal error protection transmission. This will add further support to the current considerations by the standardisation committee on adding scalability within the H.264 specification. 


\section{ACKNOWLEDGMENT}

This work has been supported by the Engineering and Physical Sciences Research Council (EPSRC) of the UK.

\section{REFERENCES}

[1] C. E. Shannon, "A mathematical theory of communication," The Bell Systems Technical Journal, vol. 27, pp. 379-423, 623656, 1948.

[2] A. J. Goldsmith and S.-G. Chua, "Adaptive coded modulation for fading channels," IEEE Transactions on Communications, vol. 46, no. 5, pp. 595-602, 1998.

[3] L. Cheng, W. Zhang, and L. Chen, "Rate-distortion optimized unequal loss protection for FGS compressed video," IEEE Transactions on Broadcasting, vol. 50, no. 2, pp. 126-131, 2004.

[4] M. Gallant and F. Kossentini, "Rate-distortion optimized layered coding with unequal error protection for robust internet video," IEEE Transactions on Circuits and Systems for Video Technology, vol. 11, no. 3, pp. 357-372, 2001.

[5] ITU-T, "Advanced video coding for generic audiovisual services," ITU-T Recommendation H.264, May 2003.

[6] ETSI, "Digital video broadcasting (DVB); framing structure, channel coding and modulation for digital terrestrial television," EN 300 744, V1.4.1, 2001.

[7] B. Barmada, M. M. Ghandi, M. Ghanbari, and E. V. Jones, "Prioritized transmission of data partitioned H.264 video with hierarchical QAM," IEEE Signal Processing Letters, vol. 12, no. 8, pp. 577-580, 2005.

[8] M. M. Ghandi and M. Ghanbari, "Layered H.264 video transmission with hierarchical QAM," Journal on Visual Communication and Image Representation, vol. 17, no. 2, pp. 451-466, 2006.

[9] A. R. Vitthaladevuni and M. S. Alouini, "A recursive algorithm for the exact BER computation of generalized hierarchical QAM constellations," IEEE Transactions on Information Theory, vol. 49, no. 1, pp. 297-307, 2003.

[10] M. Ghanbari, Standard Codecs: Image Compression to Advanced Video Coding, IEE, London, UK, 2003.

[11] T. Keller, M. Munster, and L. Hanzo, "Turbo-coded burst-byburst adaptive wide-band speech transceiver," IEEE Journal on Selected Areas in Communications, vol. 18, no. 11, pp. 2362 2372, 2000.

[12] C. Berrou and A. Glavieux, "Near optimum error correcting coding and decoding: turbo-codes," IEEE Transactions on Communications, vol. 44, no. 10, pp. 1261-1271, 1996.

[13] B. Barmada and E. V. Jones, "Adaptive modulation and coding for multimedia services," in Proceedings of the 5th IEE International Conference on $3 G$ Mobile Communication Technologies (3G '04), vol. 503, pp. 322-326, London, UK, October 2004.

M. M. Ghandi received his B.S. (1998) and M.S. (2001) degrees in electronics engineering from the University of Tehran. After two years of industrial experience in image and video coding, he joined the Video Networking Group at the University of Essex in 2003 as a Senior Research Officer where he published more than 20 papers in the field of video communications. He was granted a Ph.D. degree from this university in Febru-

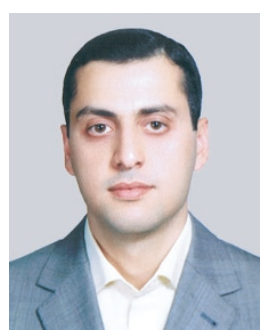
ary 2006. Recently, he took up the post of Hardware Multimedia
Design Engineer at 4i2i Communications in Aberdeen, Scotland. His research interests include reliable image and video transmission, advanced multimedia codecs, and video transcoding.

B. Barmada graduated from the University of Aleppo, Syria, in 1995 with a B.Eng. degree in computer engineering and with distinction. He received his M.S. and Ph.D. degrees from University of Essex, UK, in computer and information networks (2000) and layered image and video wireless transmission (2005), respectively. Currently he is a Lecturer at the University of Aleppo, Department of Communications. His research interests include adaptive OFDM systems, layered wireless transmission, and MIMO.

E. V. Jones started his research career with GEC Research Laboratories later transferring to the Marconi Research Laboratories. After several years of industrial telecommunications research, specialising in highcapacity transmission systems and networks, he joined the Department of Electronic Systems Engineering at the University of Essex where he is now a Senior Lecturer. His current research interests include network topologies, cellular radio network design, and adaptive modulation and coding for efficient digital transmission.

M. Ghanbari is a Professor of Video Networking in the Department of Electronic Systems Engineering, University of Essex, United Kingdom. He is best known for the pioneering work on two-layer video coding for ATM networks, now is known as SNR scalability in the standard video codecs, which earned him the Fellowship of IEEE in 2001. He has registered for eleven international patents and published more than 300

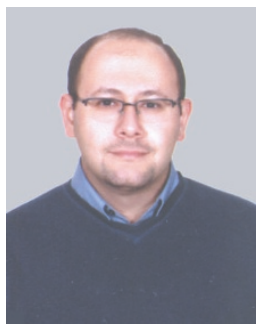
technical papers on various aspects of video networking and is the author of three books. His Video Coding: An Introduction to Standard Codecs book received the Rayleigh prize as the best book of year 2000 by IEE. His recent book Standard Codecs: Image Compression to Advanced Video Coding was published by IEE in 2003. He has been an organizing member of several international conferences and workshops. He was the General Chair of 1997 International Workshop on Packet Video and Guest Editor to 1997 IEEE Transactions on Circuits and Systems for Video Technology, Special issue on Multimedia Technology and Applications. He has served as Associate Editor to IEEE Transactions on Multimedia (IEEETMM from 1998-2004). He is a Fellow of IEEE, Fellow of IEE, and Charted Engineer (C.Eng.). 